\title{
AARP
}

\section{There is More to Perimenopause than Hot Flashes: Annotated Questionnaire}

\section{Methods}

\begin{tabular}{|c|c|}
\hline Survey sponsor & AARP \\
\hline Survey/Data collection supplier & AARP Proprietary Panel \\
\hline Population represented & U.S. women ages $35+$ \\
\hline Sample size & $\begin{array}{l}\text { 1,400. Targeted quotas were set for age, Census } \\
\text { region, marital status, race/ethnicity, and income. }\end{array}$ \\
\hline Mode of data collection & Online panel \\
\hline $\begin{array}{l}\text { Type of sample (probability/non- } \\
\text { probability) }\end{array}$ & Non-Probability \\
\hline $\begin{array}{l}\text { Start and end dates of data } \\
\text { collection }\end{array}$ & September 24 - October 4, 2020 \\
\hline $\begin{array}{l}\text { Margin of sampling error for total } \\
\text { sample }\end{array}$ & Not applicable \\
\hline $\begin{array}{l}\text { Margin of sampling error for key } \\
\text { subgroups }\end{array}$ & Not applicable \\
\hline Weighting & $\begin{array}{l}\text { The data are weighted to the U.S. population by age, } \\
\text { race, Hispanic ethnicity, marital status, census } \\
\text { division, and income to benchmarks from the Current } \\
\text { Population Survey (CPS). }\end{array}$ \\
\hline Contact for more information & $\begin{array}{l}\text { Colette Thayer, Ph.D., cthayer@aarp.org } \\
\text { Cheryl Lampkin, Ph.D., clampkin@aarp.org } \\
\text { For media inquiries, contact media@aarp.org }\end{array}$ \\
\hline
\end{tabular}




\section{Screening Questions}

S1. Are you?

\begin{tabular}{|l|c|}
\hline Male & - \\
Female & $100 \%$ \\
Identify differently & - \\
Refused & - \\
\hline
\end{tabular}

S2. What is your age?

\begin{tabular}{|l|c|}
\hline Under 35 & - \\
$35-39$ & $9 \%$ \\
$40-49$ & $30 \%$ \\
$50-59$ & $31 \%$ \\
$60-69$ & $16 \%$ \\
$70+$ & $16 \%$ \\
\hline
\end{tabular}

S3. Which of the following best describes your race? Select all that apply

\begin{tabular}{|l|c|}
\hline American Indian or Alaska Native & $1 \%$ \\
Asian & $4 \%$ \\
Black or African American & $14 \%$ \\
Native Hawaiian or Other Pacific Islander & $<0.5 \%$ \\
White & $79 \%$ \\
Other race & $4 \%$ \\
Refused & - \\
\hline
\end{tabular}

S4: Are you of Spanish, Hispanic or Latino background?

\begin{tabular}{|l|c|}
\hline Yes & $15 \%$ \\
No & $85 \%$ \\
Refused & - \\
\hline
\end{tabular}




\section{AARP'}

S5. What is your current marital / relationship status?

\begin{tabular}{|l|c|}
\hline Married & $62 \%$ \\
Living with a partner & $5 \%$ \\
Widowed & $8 \%$ \\
Divorced or Separated & $13 \%$ \\
Never Married & $13 \%$ \\
Refused & - \\
\hline
\end{tabular}

S6. What was your household's total income from all sources in 2019?

\begin{tabular}{|l|c|}
\hline Less than $\$ 25,000$ & $14 \%$ \\
$\$ 25,000$ to less than $\$ 50,000$ & $21 \%$ \\
$\$ 50,000$ to less than $\$ 75,000$ & $16 \%$ \\
$\$ 75,000$ to less than $\$ 100,000$ & $16 \%$ \\
$\$ 100,000$ to less than $\$ 150,000$ & $19 \%$ \\
$\$ 150,000$ to less than $\$ 200,000$ & $7 \%$ \\
$\$ 200,000$ or more & $7 \%$ \\
Prefer not to provide & - \\
Refused & - \\
\hline
\end{tabular}

S7. What is your zip code? Please enter your 5 digit zip code. 
Main

Q1: In general, how would you rate your physical health?

\begin{tabular}{|l|c|}
\hline Top 2 Box (Net) & $43 \%$ \\
Excellent (5) & $10 \%$ \\
Very Good (4) & $33 \%$ \\
Good (3) & $39 \%$ \\
Bottom 2 Box (Net) & $18 \%$ \\
Fair (2) & $15 \%$ \\
Poor (1) & $3 \%$ \\
\hline
\end{tabular}

Q2: In general, how would you rate your mental health?

\begin{tabular}{|l|c|}
\hline Top 2 Box (Net) & $56 \%$ \\
Excellent (5) & $23 \%$ \\
Very Good (4) & $33 \%$ \\
Good (3) & $30 \%$ \\
Bottom 2 Box (Net) & $15 \%$ \\
Fair (2) & $12 \%$ \\
Poor (1) & $3 \%$ \\
\hline
\end{tabular}


Q3: Have you experienced any of the following?

\begin{tabular}{|c|c|c|c|c|}
\hline & $\begin{array}{c}\text { Yes } \\
\text { (Net) }\end{array}$ & $\begin{array}{c}\text { Yes - } \\
\text { In the past } \\
\text { year }\end{array}$ & $\begin{array}{c}\text { Yes - } \\
\text { More than } \\
\text { a year ago }\end{array}$ & No \\
\hline Sleep problems & $51 \%$ & $41 \%$ & $10 \%$ & $49 \%$ \\
\hline Skin dryness & $46 \%$ & $37 \%$ & $9 \%$ & $54 \%$ \\
\hline Fatigue/tired (more so than usual) & $45 \%$ & $36 \%$ & $9 \%$ & $55 \%$ \\
\hline Weight gain and slowed metabolism & $45 \%$ & $32 \%$ & $13 \%$ & $55 \%$ \\
\hline Skin sagging/looseness & $39 \%$ & $29 \%$ & $10 \%$ & $61 \%$ \\
\hline Anxiety/panic & $36 \%$ & $29 \%$ & $8 \%$ & $64 \%$ \\
\hline Mood swings/irritability & $34 \%$ & $26 \%$ & $8 \%$ & $66 \%$ \\
\hline Hair thinning or loss & $33 \%$ & $22 \%$ & $11 \%$ & $67 \%$ \\
\hline Depression & $33 \%$ & $24 \%$ & $9 \%$ & $67 \%$ \\
\hline Increased facial hair growth (on upper lip, chin, or jaw line) & $33 \%$ & $16 \%$ & $16 \%$ & $67 \%$ \\
\hline Reduced libido or sex drive & $31 \%$ & $20 \%$ & $10 \%$ & $69 \%$ \\
\hline Hot flashes & $31 \%$ & $22 \%$ & $9 \%$ & $69 \%$ \\
\hline Night sweat & $30 \%$ & $22 \%$ & $8 \%$ & $70 \%$ \\
\hline Migraines or frequent headaches & $30 \%$ & $22 \%$ & $8 \%$ & $70 \%$ \\
\hline Mental fog/difficulty concentrating & $29 \%$ & $22 \%$ & $7 \%$ & $71 \%$ \\
\hline Heart palpitations (fast-beating, fluttering or pounding heart) & $29 \%$ & $18 \%$ & $12 \%$ & $71 \%$ \\
\hline Vaginal dryness & $26 \%$ & $19 \%$ & $7 \%$ & $74 \%$ \\
\hline Itchy/crawly skin & $24 \%$ & $19 \%$ & $5 \%$ & $76 \%$ \\
\hline Incontinence (lack urination or bowel/stool control) & $21 \%$ & $14 \%$ & $7 \%$ & $79 \%$ \\
\hline Acne & $21 \%$ & $13 \%$ & $8 \%$ & $79 \%$ \\
\hline Tingling extremities & $20 \%$ & $16 \%$ & $5 \%$ & $80 \%$ \\
\hline Changes in menstruation & $20 \%$ & $12 \%$ & $9 \%$ & $80 \%$ \\
\hline Difficulty achieving orgasm & $18 \%$ & $13 \%$ & $6 \%$ & $82 \%$ \\
\hline Breast tenderness & $17 \%$ & $12 \%$ & $4 \%$ & $84 \%$ \\
\hline Painful intercourse & $17 \%$ & $10 \%$ & $7 \%$ & $83 \%$ \\
\hline Extremely heavy periods & $16 \%$ & $9 \%$ & $7 \%$ & $84 \%$ \\
\hline Burning tongue & $4 \%$ & $2 \%$ & $2 \%$ & $96 \%$ \\
\hline Increased hair growth on other areas of body & $11 \%$ & $7 \%$ & $4 \%$ & $89 \%$ \\
\hline Median (Mean) & $6(8)$ & & & \\
\hline
\end{tabular}

Q4: What have you done in response to these changes in your body? [OPEN-ENDED] 
Q5: Could any of the following result from a decline in women's hormone levels as they age, or are you not sure?

\begin{tabular}{|c|c|c|c|}
\hline & Yes & No & Not sure \\
\hline Hot flashes & $63 \%$ & $21 \%$ & $16 \%$ \\
\hline Weight gain and slowed metabolism & $62 \%$ & $21 \%$ & $17 \%$ \\
\hline Night sweat & $59 \%$ & $23 \%$ & $18 \%$ \\
\hline Vaginal dryness & $59 \%$ & $23 \%$ & $18 \%$ \\
\hline Changes in menstruation & $58 \%$ & $26 \%$ & $16 \%$ \\
\hline Reduced libido or sex drive & $56 \%$ & $24 \%$ & $20 \%$ \\
\hline Mood swings/irritability & $55 \%$ & $23 \%$ & $22 \%$ \\
\hline Hair thinning or loss & $55 \%$ & $24 \%$ & $22 \%$ \\
\hline Fatigue/tired (more so than usual) & $53 \%$ & $23 \%$ & $25 \%$ \\
\hline Increased facial hair growth (on upper lip, chin, or jaw line) & $53 \%$ & $25 \%$ & $21 \%$ \\
\hline Sleep problems & $51 \%$ & $22 \%$ & $27 \%$ \\
\hline Skin sagging/looseness & $49 \%$ & $25 \%$ & $26 \%$ \\
\hline Painful intercourse & $48 \%$ & $27 \%$ & $26 \%$ \\
\hline Skin dryness & $48 \%$ & $23 \%$ & $30 \%$ \\
\hline Difficulty achieving orgasm & $47 \%$ & $26 \%$ & $27 \%$ \\
\hline Mental fog/difficulty concentrating & $44 \%$ & $28 \%$ & $28 \%$ \\
\hline Depression & $43 \%$ & $28 \%$ & $29 \%$ \\
\hline Increased hair growth on other areas of body & $43 \%$ & $28 \%$ & $29 \%$ \\
\hline Incontinence (lack urination or bowel/stool control) & $39 \%$ & $30 \%$ & $31 \%$ \\
\hline Breast tenderness & $37 \%$ & $31 \%$ & $33 \%$ \\
\hline Anxiety/panic & $35 \%$ & $30 \%$ & $35 \%$ \\
\hline Extremely heavy periods & $34 \%$ & $34 \%$ & $31 \%$ \\
\hline Migraines or frequent headaches & $32 \%$ & $31 \%$ & $37 \%$ \\
\hline High blood pressure [DECOY] & $32 \%$ & $32 \%$ & $36 \%$ \\
\hline Acne & $31 \%$ & $35 \%$ & $35 \%$ \\
\hline Heart palpitations (fast-beating, fluttering or pounding heart) & $29 \%$ & $33 \%$ & $38 \%$ \\
\hline Hearing loss [DECOY] & $27 \%$ & $40 \%$ & $33 \%$ \\
\hline Itchy/crawly skin & $25 \%$ & $31 \%$ & $44 \%$ \\
\hline Type 2 Diabetes [DECOY] & $23 \%$ & $38 \%$ & $39 \%$ \\
\hline Tingling extremities & $21 \%$ & $33 \%$ & $47 \%$ \\
\hline Burning tongue & $13 \%$ & $38 \%$ & $49 \%$ \\
\hline
\end{tabular}




\section{AARP'}

Q6: How informed do you feel about changes in your body that could result from a decline in your hormone levels as you age?

\begin{tabular}{|c|c|}
\hline Top 2 Box (Net) & $69 \%$ \\
4 - Very informed (4) & $18 \%$ \\
3 - Somewhat informed (3) & $51 \%$ \\
Bottom 2 Box (Net) & $31 \%$ \\
2 - Not too informed (2) & $24 \%$ \\
1 - Not at all informed (1) & $7 \%$ \\
\hline
\end{tabular}

Q7. How, if at all, have or will changes in your body that result from a decline in your hormone levels as you age impact how you view yourself? Have or will they make you feel...

\begin{tabular}{|l|c|}
\hline Old & $40 \%$ \\
Like I am starting a new phase in my life & $40 \%$ \\
Human & $26 \%$ \\
More mature & $24 \%$ \\
Less feminine & $15 \%$ \\
Ugly & $12 \%$ \\
Free & $10 \%$ \\
Happy & $8 \%$ \\
Empowered & $6 \%$ \\
Invisible & $5 \%$ \\
More feminine & $5 \%$ \\
Fallible & $5 \%$ \\
More beautiful & $4 \%$ \\
Renewed & $4 \%$ \\
None of the above & $22 \%$ \\
\hline
\end{tabular}

Q8: Do you feel what happens to women as they experience declining hormone levels as they age is discussed...

\begin{tabular}{|l|l|}
\hline Too little & $57 \%$ \\
The right amount & $33 \%$ \\
Too much & $11 \%$ \\
\hline
\end{tabular}

Q9: Do you feel the physical impact of declining hormone levels in women as they age is discussed...

\begin{tabular}{|l|l|}
\hline Too little & $58 \%$ \\
The right amount & $32 \%$ \\
Too much & $11 \%$ \\
\hline
\end{tabular}


Q10: Do you feel the mental impact of declining hormone levels in women as they age is discussed...

\begin{tabular}{|l|l|}
\hline Too little & $61 \%$ \\
The right amount & $29 \%$ \\
Too much & $10 \%$ \\
\hline
\end{tabular}

Q11: What do you think the average age is that hormone levels start to decline in a woman's body as she ages?

\begin{tabular}{|l|c|}
\hline $30-34$ & $2 \%$ \\
$35-39$ & $11 \%$ \\
$40-44$ & $22 \%$ \\
$45-49$ & $25 \%$ \\
$50-54$ & $21 \%$ \\
55 to & $9 \%$ \\
60 or older & $5 \%$ \\
Not sure & $5 \%$ \\
\hline
\end{tabular}

Q12: Have you ever discussed how declining hormone levels impact a woman's body as she ages with...

\begin{tabular}{|l|l|}
\hline A friend & $41 \%$ \\
\hline Your mother or mother figure & $25 \%$ \\
\hline A relative other than your mother or mother figure & $28 \%$ \\
\hline A doctor or other healthcare provider & $45 \%$ \\
\hline
\end{tabular}

Q13 Did you find the conversation with [INSERT FROM PREVIOUS QUESTION] about declining hormone levels...

\begin{tabular}{|c|c|c|c|c|c|c|}
\hline & $\begin{array}{c}\text { Top } \\
2 \text { Box } \\
\text { (Net) }\end{array}$ & $\begin{array}{c}\text { Very } \\
\text { helpful } \\
(4)\end{array}$ & $\begin{array}{c}\text { Somewhat } \\
\text { Helpful } \\
\text { (3) }\end{array}$ & $\begin{array}{c}\text { Bottom } \\
2 \text { Box } \\
\text { (Net) }\end{array}$ & $\begin{array}{c}\text { Not too } \\
\text { helpful } \\
\text { (2) }\end{array}$ & $\begin{array}{c}\text { Not at all } \\
\text { helpful } \\
(1)\end{array}$ \\
\hline A friend $(n=565)$ & $85 \%$ & $23 \%$ & $63 \%$ & $15 \%$ & $14 \%$ & $1 \%$ \\
\hline $\begin{array}{l}\text { Your mother or mother figure } \\
(n=341)\end{array}$ & $86 \%$ & $31 \%$ & $55 \%$ & $14 \%$ & $11 \%$ & $3 \%$ \\
\hline $\begin{array}{l}\text { A relative other than your mother } \\
\text { or mother figure }(n=381)\end{array}$ & $83 \%$ & $25 \%$ & $58 \%$ & $17 \%$ & $14 \%$ & $3 \%$ \\
\hline $\begin{array}{l}\text { A doctor or other healthcare } \\
\text { provider }(n=622)\end{array}$ & $90 \%$ & $38 \%$ & $52 \%$ & $10 \%$ & $8 \%$ & $2 \%$ \\
\hline
\end{tabular}




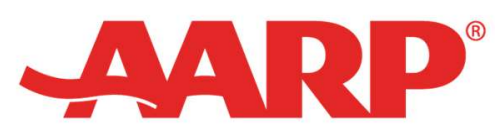

Q14: Have you ever searched for information online about how declining hormone levels impact a woman's body as she ages?

\begin{tabular}{|l|l|}
\hline Yes & $31 \%$ \\
No & $69 \%$ \\
\hline
\end{tabular}

Q15: Have you ever searched for information online about how perimenopause or menopause impacts a woman's body as she ages?

\begin{tabular}{|l|l|}
\hline Yes & $37 \%$ \\
No & $63 \%$ \\
\hline
\end{tabular}

